\title{
A More Accurate Approach to Amyloid Detection and Subtyping: Combining in situ Congo Red Staining and Immunohistochemistry
}

\author{
Thomas Menter ${ }^{\mathrm{a}, \mathrm{b}} \quad$ Matthias Bachmann ${ }^{\mathrm{a}}$ Susanne Grieshaber ${ }^{\mathrm{a}}$ \\ Alexandar Tzankov ${ }^{a}$ \\ ${ }^{a}$ Institute of Pathology, University Hospital Basel, Basel, Switzerland; ${ }^{b}$ Department of Histopathology, Hammersmith \\ Hospital Campus, Imperial College Healthcare NHS Trust, London, UK
}

\section{Key Words}

Amyloidosis · Congo red $\cdot$ Immunohistochemistry $\cdot$ Double staining $\cdot$ Fluorescence

\begin{abstract}
Amyloidosis is the result of various, differently approachable diseases. It is vital to subtype the amyloid deposits in order to establish and finally treat the underlying disease properly. Besides the classical staining with Congo red, further procedures like immunohistochemical staining are needed for classification. Here, we present a more accurate approach using Congo red/immunohistochemical double staining easily applicable in routine diagnostics. Modifications of the Congo red staining technique and the immunohistochemical procedures were needed in order to combine both staining procedures on one slide. The evaluation was done using conventional light and fluorescence microscopy. By shortening the staining time for Congo red to $10 \mathrm{~s}$ and by modification regarding endogenous peroxidase blockage, accurate results could be obtained for evaluating the Congo red/immunohistochemistry double staining using a fluorescence microscope. Sections of $2 \mu \mathrm{m}$ instead of $4 \mu \mathrm{m}$ thickness were superior for evaluation, since they increased staining speci-
\end{abstract}

\section{KARGER}

(C) 2016 S. Karger AG, Basel

E-Mail karger@karger.com

www.karger.com/pat ficity. The combination of Congo red and immunohistochemistry as in situ double staining on one slide is a feasible approach in the diagnosis of amyloidosis. It allows focusing on the fluorescent Congo red-positive areas when evaluating immunohistochemistry, thus avoiding signing out falsepositive results. Additionally, it increases the signal-to-noise ratio of the immunohistochemically stained sections on conventional microscopy.

(c) 2016 S. Karger AG, Basel

\section{Introduction}

Amyloidosis is the morphological description of the deposition of wrongly folded proteins that can be found in many different tissue types [1]. The proteins forming amyloid are derived from a plethora of sources, ranging from tumors, like plasma cell neoplasms or medullary thyroid carcinomas, to chronic infections, or are seen as a result of aging. In rare cases, amyloidosis is due to genetically inherited protein misfolding disorders [2]. The clinical importance of amyloidosis reaches from incidental finding at autopsies to life-threatening diseases. When examining tissue specimens it is important for patholo- 
gists to keep the likeliness of amyloid deposits in mind as this might be the first clue for a yet unknown serious underlying disease. Importantly, although there are promising results regarding therapies targeting the amyloid deposits themselves [3], treatment approaches in amyloidosis still mainly aim at the underlying disorder [4] and, in the majority of cases, this, in turn, can and must be determined based on amyloid subtypization.

The classical histochemical staining for amyloid is Congo red, as introduced by Bennhold in 1922 [5]; the typical 'apple green' birefringence using polarization was first described in 1927 [6]. In order to increase the sensitivity and specificity of Congo red staining, several methods have been used including evaluation of Congo red-stained sections by fluorescent light because of the known fluorescent properties of Congo red [7-9]. Just recently, approaches using a digitally reinforced hematoxylin-eosin polarization technique have been proposed for amyloid detection $[10,11]$, being particularly helpful in identifying amyloid in cases with scanty tissue available.

The gold standard for subtyping amyloid is immunohistochemistry, including several well-established protocols [12]; however, there are some shortcomings, especially considering the poorer specificity of antibodies against amyloidogenic light chain lambda. A new approach to subtyping especially rare variants of amyloidosis is mass spectrometry [13]; unfortunately, due to cost and availability issues, this promising method has not been widely used so far, with an additional shortcoming being its obvious limitation in cases with scanty tissue available or with limited amount of deposed amyloid. Another recent achievement is the use of immunoelectron microscopy of abdominal fat aspirates for subtyping amyloid deposits [14]; however, this is a sophisticated method.

Several earlier studies have focused on the combination of fluorescence and immunohistochemistry [7, 15, 16]. Here, we present additional evidence for the practicability of such an approach and report a protocol easily translatable into routine, which enabled us to accurately address amyloid subtypization on formalin-fixed and paraffin-embedded tissues. Congo red/immunohistochemistry double staining allows focusing on the Congo red-positive areas when evaluating immunohistochemistry and increases the signal-to-noise ratio of the immunohistochemically stained sections on conventional light microscopic evaluation, improving both the specificity and sensitivity of the procedure as well as reducing the amount of tissue needed for diagnostics.
Table 1. Details of tissues used for establishing the combined in situ Congo red and immunohistochemistry staining

\begin{tabular}{lll}
\hline $\begin{array}{l}\text { Amyloid } \\
\text { subtype }\end{array}$ & Tissue and patient details & $\begin{array}{l}\text { Steps }{ }^{1} \text { the tissue } \\
\text { was used for }\end{array}$ \\
\hline AA & Heart, senile amyloidosis & 1,2 \\
AL lambda & Heart, known multiple myeloma & 1,2 \\
AL lambda & Small salivary gland & 2,3 \\
AL lambda & Bone marrow (3 cases) & 3 \\
AL kappa & Bone marrow (4 cases) & 3 \\
AL kappa & Oral mucosa, amyloid tumor & 3 \\
AL lambda & Gastric mucosa & 3 \\
\hline
\end{tabular}

$\mathrm{AA}=$ amyloid $\mathrm{A} ; \mathrm{AL}=$ light chain .

${ }^{1}$ Steps: 1 - modifications of the Congo red staining protocol; 2 - modifications of the immunohistochemical protocol for combined Congo red and immunohistochemical staining on one slide; 3 - modifications of thickness of sections.

\section{Materials and Methods}

\section{Selection of Cases}

Specimens with a proven diagnosis of amyloidosis from our archive were used to establish the new technique. They are listed in table 1 including the steps the respective tissue was used for.

\section{Congo Red Staining Approaches}

Congo red staining was initially performed according to the established protocol at our institute: formalin-fixed and paraffinembedded tissue is cut to $6-\mu \mathrm{m}$-thick sections, deparaffinized and dyed with Congo red for $30 \mathrm{~min}-2.5 \mathrm{~g}$ of Congo red (Merck Millipore, Darmstadt, Germany) and 1 g potassium hydroxide (Merck Millipore) dissolved in $500 \mathrm{ml}$ of $80 \%$ ethanol; after that, sections are rinsed with tap water before they are counterstained with hematoxylin (Merck Millipore) for $4 \mathrm{~min}$. In a next step, sections are differentiated into $0.5 \% \mathrm{HCl} /$ ethanol solution and rinsed again with tap water for $10 \mathrm{~min}$ before they are dehydrated and mounted.

For our study, the protocol was modified regarding the Congo red staining time in order to see, which impact shorter staining times might have on the subsequent immunohistochemical staining. Sections with a thickness of 2 and $4 \mu \mathrm{m}$ were tested as well.

\section{Immunohistochemical Staining and Endogenous Peroxidase}

\section{Blockage}

The immunohistochemical staining conditions for the various amyloid subtypes are listed in table 2 . As suggested by the manufacturer, two different clones were used for the detection of the amyloidogenic light chains kappa and lambda, respectively. Immunohistochemistry was performed after the Congo red staining procedure. Briefly, deparaffinized and endogenous peroxidase blocked slides (see later) were incubated in normal horse (for AA amyloid staining) or normal goat serum (for all other stainings)/ Tris-buffered saline (TBS) solution $(900 \mu \mathrm{l}$ serum/60 ml TBS) for $30 \mathrm{~min}$ at room temperature (both sera from Vector Lab., Burlingame, Calif., USA) and then incubated overnight with the primary 
Table 2. Antibodies used for immunohistochemical staining of amyloid subtypes

\begin{tabular}{lllll}
\hline & Source & Dilution, incubation & Pretreatment & Detection \\
\hline Amyloid A & AmYmed, clone mcC & prediluted (1:30), overnight & none & AEC \\
Amyloid ATTR & AmYmed, clone TIE & prediluted (1:800), overnight & none & AEC \\
Amyloid kappa & AmYmed, clone SIN & prediluted $(1: 1,000)$, overnight & none & AEC \\
Amyloid kappa & AmYmed, clone KRA/KUN & prediluted $(1: 2,000)$, overnight & none & AEC \\
Amyloid lambda & AmYmed, clone HAR & prediluted $(1: 1,000)$, overnight & none & AEC \\
Amyloid lambda & AmYmed, clone ULI/LAT & prediluted (1:500), overnight & none & AEC \\
\hline
\end{tabular}

antibody at $4{ }^{\circ} \mathrm{C}$, rinsed and incubated with biotinylated antimouse IgG (for amyloid AA staining) or biotinylated anti-rabbit IgG (for all other staining procedures) solution $(300 \mu \mathrm{lgG} / 900 \mu \mathrm{l}$ normal horse or goat serum/60 ml TBS; both IgG form Vector Lab.) for $30 \mathrm{~min}$ at room temperature; finally, they were rinsed and incubated with an avidin-biotin-peroxidase kit (Vectastain from Vector Lab.) for $30 \mathrm{~min}$ and visualized applying ready-to-use 3-amino-9-ethylcarbazole (AEC; from Dako, Glostrup, Denmark) as chromogen (10-20 min under instantaneous optical control) and Meyer's hematoxylin (from Medite, Dietikon, Switzerland). AEC was preferred since previous experiments showed better signal-to-noise results for AEC visualization compared to diaminobenzidine visualization. As AEC is water and alcohol soluble, the slides were covered with Medi-Mount (from Medite, Burgdorf, Germany) for 30 min before coverslipping.

Since the usual treatment for endogenous peroxidase blockage of tissues $\left(3 \% \mathrm{H}_{2} \mathrm{O}_{2}\right.$ solution in $70 \%$ ethanol) after completing the Congo red stain led to loss of congophilia, further methods for endogenous peroxidase blockage were tested, such as: moving this step prior to the Congo red staining and substitution of $70 \%$ ethanol by distilled water.

\section{Evaluation}

The Congo red/immunohistochemistry double-stained sections were primarily evaluated using a conventional light microscope to estimate if the respective immunohistochemical chromogen product was visualized as expected, worse or better compared to the diagnostic routine staining (without Congo red prestaining). Subsequently, a fluorescence microscope with a rhodamine filter (Zeiss Axioskop 40; Zeiss, Göttingen, Germany) was used to evaluate the fluorescence of the Congo red dye. Areas that showed specific intensive fluorescence were further evaluated by light microscopy to see whether amyloid deposits could also be detected by immunohistochemistry in these areas.

\section{Results}

\section{Step 1: Modifications of the Congo Red Staining Protocol}

Several staining times for the Congo red dye were tested. This was necessary as the ability of antibodies to bind antigens was reduced by the Congo red dying pro- cedure and full-intensity Congo red made concomitant evaluation difficult, since a red chromogen (AEC) with a similar color as that of Congo red was used for immunohistochemistry. Applying the original staining time of $30 \mathrm{~min}$, birefringence could be detected for amyloid deposits using a polarized filter on conventional light microscopy. When staining for Congo red lasted 5,10 or 20 s only, positive signals could always be detected when using the rhodamine filter of a fluorescence microscope, while conventional microscopy only occasionally showed congophilia and birefringence using the polarized filter (fig. 1). For further analysis, Congo red staining time was preferably reduced to $10 \mathrm{~s}$ in order to avoid overstaining; $10 \mathrm{~s}$ has been chosen for practicability reasons (easier to steadily comply compared to $5 \mathrm{~s}$ ) in the wet lab.

\section{Step 2: Modifications of the Immunohistochemical}

Protocols for Combining Congo Red and

Immunohistochemical Staining on One Slide

In a first step, immunohistochemical staining was performed on separate sections to confirm antigenicity of the tissues used for this study, which showed satisfactory staining results using the established routine protocols as detailed in table 2.

When combining Congo red staining (Congo red staining time of $10 \mathrm{~s}$ ) with immunohistochemical staining on the same sections, it was noted that congophilia disappeared after completing the immunohistochemical procedures using the common treatment for endogenous peroxidase blockage of tissues $\left(3 \% \mathrm{H}_{2} \mathrm{O}_{2}\right.$ solution in $70 \%$ ethanol) since the Congo red dye has been washed out. Therefore, in a third step, the treatment for endogenous peroxidase blockage was modified. Endogenous peroxidase blockage using the conventional method but applying it prior to the Congo red staining procedure as well as substitution of the $70 \%$ ethanol by distilled water, when performing endogenous peroxidase blockage after 


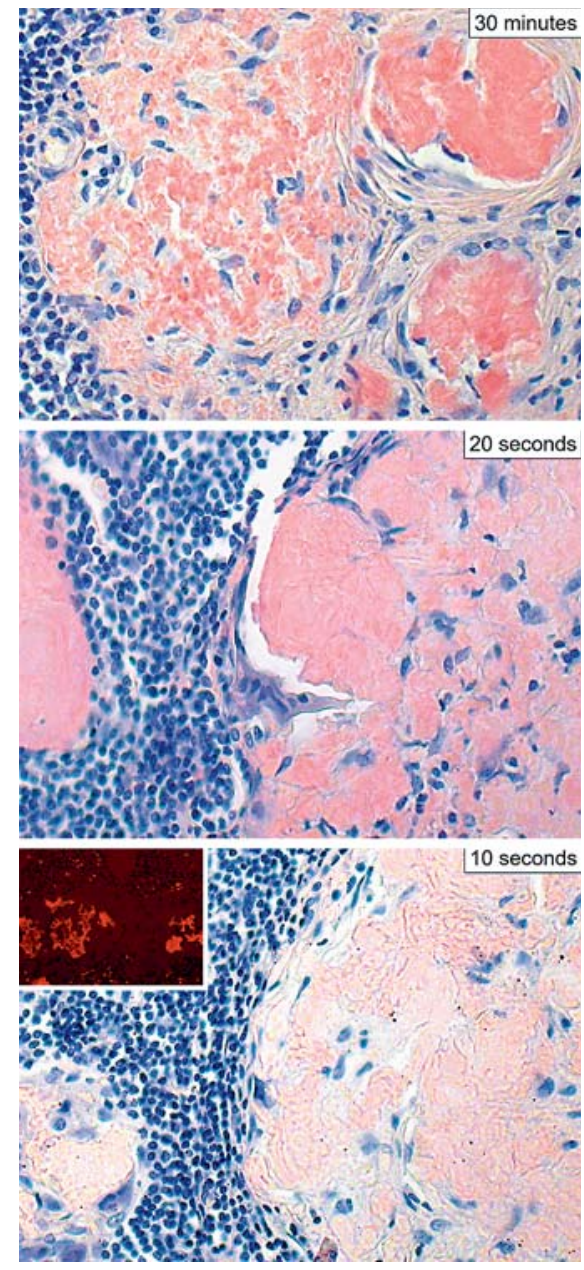

Fig. 1. Staining results of Congo red using staining times of $30 \mathrm{~min}$, 20 and $10 \mathrm{~s}$, respectively. The intensity of Congo red staining gets clearly diminished due to the reduced staining time, yet fluorescence of Congo red is still readily detectable even at a staining time of $10 \mathrm{~s}$ (see inset).

Congo red staining, led to satisfactory results, both for the evaluation of Congo red staining and for immunohistochemical staining. In order to stick to the usual wet lab workflow as much as possible, the method substituting ethanol by distilled water was further applied. Attempting to omit endogenous peroxidase blockage moderately decreased the signal-to-noise results for AEC visualization (assuming that the unblocked endogenous enzymes utilized AEC and, on the one hand, slightly background-stained and, on the other, deprived the $\mathrm{ABC}$-peroxidase complex of chromogen for proper visualization) and was therefore not further exploited. The quality of immunohistochemical staining after prestain- ing with Congo red was the same as that of immunohistochemical staining without previous Congo red staining; thus, we concluded that performing immunohistochemistry on sections prestained with Congo red for $10 \mathrm{~s}$ does not lead to a loss of antigenicity or to unspecific staining results.

\section{Step 3: Modifications of Section Thicknesses}

Finally, we examined the role of different section thicknesses ( 2 and $4 \mu \mathrm{m}$ ). In general, the specificity and evaluability of the immunohistochemical staining (signal-tonoise ratio) was better on the thinner sections; besides, there were fewer artifacts regarding the detachment of tissue from the slides during the staining procedures. All cases showed the same results as obtained during the initial diagnostic subtyping of amyloid deposits.

\section{Examples of Implementation of the Described}

Approach into Routine Diagnostic Procedures

Figures 2 and 3 illustrate two routine cases, in which amyloid subtypization could be achieved using the new algorithm proposed in this study; by applying the conventional technique only, no interpretable results have been obtained.

\section{Discussion}

Here, we present and validate a combined Congo red/ immunohistochemistry double staining procedure, which allows focusing on the Congo red-positive areas when evaluating immunohistochemistry, thus improving the specificity of in situ-based amyloid subtypization. It also helps sparing tissue, which is an issue as biopsy material is becoming more and more limited due to changed diagnostic approaches using fine needle cores and biopsy forcipes.

In contrast to other proposed new evaluation techniques focusing on sophisticated digitalized image evaluation $[10,11]$, our approach can be easily incorporated into routine diagnostics. Prerequisites only comprise modifying established Congo red staining, available amyloid immunohistochemistry as well as a fluorescence microscope with a rhodamine filter. Ideally, microscopes, which are equipped for both fluorescence and conventional light microscopy, should be used, as this simplifies the verification process on the same (fluorescent) areas of interest of amyloid deposition when evaluating immunohistochemistry by a simple switch of light source and filter. However, two different microscopes could be used as 
Fig. 2. Tonsil tissue of a patient with lightchain amyloidosis with underlying kapparestricted plasma cell neoplasia; both antibodies for lambda light chains stain falsely positive, but the staining distribution does not correspond to areas of Congo red fluorescence. In contrast, the areas positive for both kappa antibodies correspond to areas of Congo red fluorescence.
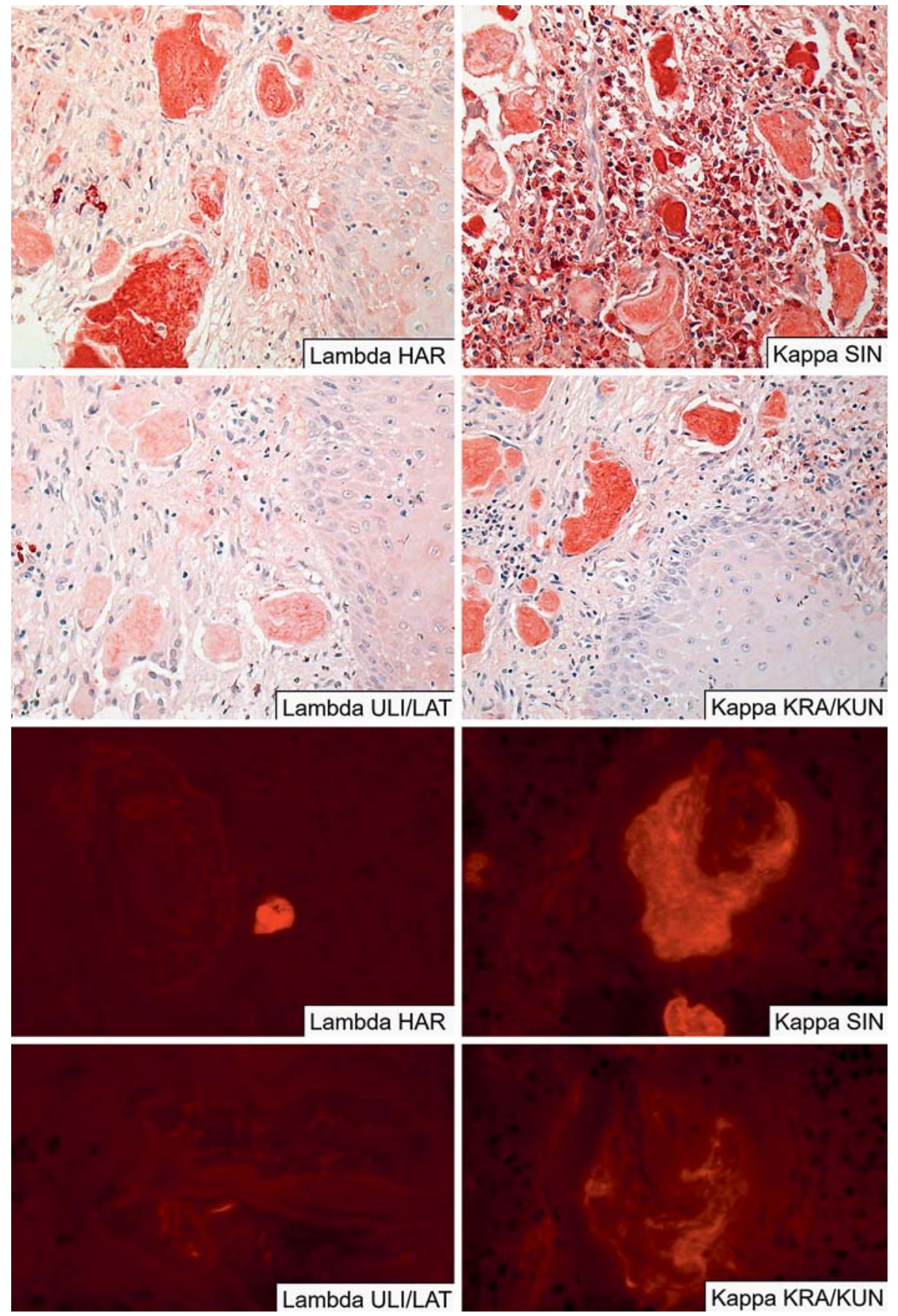

well, yet in difficult cases, taking a photo of the respective areas in fluorescence and light microscopy might be suggested for easier evaluation.

In concordance with previous studies, we could show that Congo red does not interfere with or disturb immunohistochemistry [16]. In addition, compared to section thicknesses of 4-6 $\mu \mathrm{m}$ used in most studies so far [7, 15,
16], we could demonstrate that using thinner sections of $2 \mu \mathrm{m}$, which are advantageous for immunohistochemistry (better signal-to-noise ratio, less detachment artifacts), can also lead to satisfactory results regarding Congo red staining, if read out by a fluorescent microscope.

There have been several recent papers propagating the use of fluorescence for evaluating amyloid deposits and 

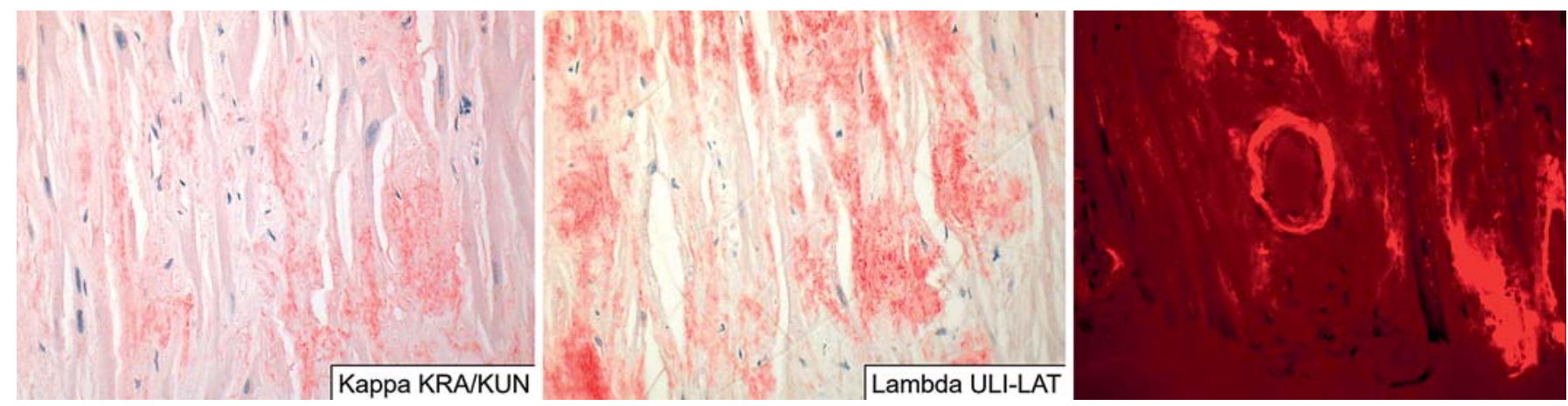

Fig. 3. Heart tissue of a patient with underlying lambda-restricted plasma cell neoplasia; both lambda antibodies stain positively, but one kappa antibody (KRA/KUN) also shows focal weak positivity. Comparison with the Congo red fluorescence (right) confirms that amyloid deposits cannot be detected in the areas of light-chain kappa positivity. These are only seen in areas staining for light-chain lambda.

demonstrating its superiority over polarized light microscopy by being both more specific and more sensitive [8, 17]. In this study, we have refined and optimized this approach in several ways by working on an adapted protocol regarding staining time, staining procedures as well as section thickness. These changes finally enabled performing an in situ double staining for both, sensitive and specific detection of amyloid deposits as well as for subtypization of these deposits, which is crucial to distinguish the underlying disease leading to amyloid deposits. Our double staining approach not only allows to sensitively detect amyloid by fluorescence and at the same time to specifically categorize exactly these deposits, but also to spare material avoiding serial sections, on which the sometimes minute amyloid deposits might have already been cut away. Since the establishment of this double staining, we have already used this approach in several cases, in which a final diagnosis could not be reached by other methods. Figures 2 and 3 illustrate two of these cases.

However, there might still be space for additional perfection. As immunoalkaline-phosphatase-based detection systems that do not need endogenous peroxidase blockage are not currently used in our immunohistochemical workflow, substitution of immunoperoxidasebased detection systems by the former may further improve results and lean the lab workflow.

To conclude, we present an optimized and refined approach for Congo red/immunohistochemistry double staining. This approach is beneficial regarding specificity, costs and amount of tissue needed. Its requirements are minimal and should allow a widespread use.

\section{Acknowledgements}

Thomas Menter is supported by the Nuovo-Soldati Cancer Research Foundation, Vaduz, Liechtenstein.

\section{Statement of Ethics}

The study only involves archive human material from the Institute of Pathology Basel and was approved by the Ethics Committee of Northwestern and Central Switzerland (EKNZ 2014252). No animals were involved.

\section{Disclosure Statement}

The authors declare that they have no conflicts of interest.

References J Haematol 2001;114:529-538.

2 Mollee P, Renaut P, Gottlieb D, Goodman H: How to diagnose amyloidosis. Intern Med J 2014;44:7-17.

3 Wechalekar AD, Gillmore JD, Hawkins PN: Systemic amyloidosis. Lancet 2015, Epub ahead of print.

-4 Kastritis E, Dimopoulos MA: Recent advances in the management of AL amyloidosis. Br J Haematol 2016;172:170-186.

5 Bennhold H: Specific staining of amyloid by Congo red. Munch Med Wochenschr 1922; 69:1537-1538.

6 Divry P, Florkin M: Sur les propriétés optiques de l'amyloide. Paris, CR Société de Biologie, 1927, pp 1808-1810. 
7 Linke RP: Highly sensitive diagnosis of amyloid and various amyloid syndromes using Congo red fluorescence. Virchows Arch 2000; 436:439-448.

$>8$ Marcus A, Sadimin E, Richardson M, Goodell L, Fyfe B: Fluorescence microscopy is superior to polarized microscopy for detecting amyloid deposits in Congo red-stained trephine bone marrow biopsy specimens. Am J Clin Pathol 2012;138:590-593.

-9 Puchtler H, Sweat F: Congo red as a stain for fluorescence microscopy of amyloid. J Histochem Cytochem 1965;13:693-694.

10 Doganavsargil B, Buberal GE, Toz H, Sarsik B, Pehlivanoglu B, Sezak M, Sen S: Digitally reinforced hematoxylin-eosin polarization technique in diagnosis of rectal amyloidosis. World J Gastroenterol 2015;21:1827-1837.
11 Sen S, Sarsik Kumbaraci B: Digitally reinforced polarization of hematoxylin-eosin in the diagnosis of renal amyloidosis. Turk $\mathrm{Pa}$ toloji Derg 2012;28:204-212.

12 Linke RP: On typing amyloidosis using immunohistochemistry. Detailled illustrations, review and note on mass spectrometry. Prog Histochem Cytochem 2012;47:61-132.

13 Loo D, Mollee PN, Renaut P, Hill MM: Proteomics in molecular diagnosis: typing of amyloidosis. J Biomed Biotechnol 2011;2011: 754109 .
4 Fernández de Larrea C, Verga L, Morbini P, Klersy C, Lavatelli F, Foli A, Obici L, Milani P, Capello GL, Paulli M, Palladini G, Merlini G: A practical approach to the diagnosis of systemic amyloidoses. Blood 2015;125:22392244.

15 Sen S, Başdemir G: Diagnosis of renal amyloidosis using Congo red fluorescence. Pathol Int 2003;53:534-538.

16 Linke RP, Gärtner HV, Michels H: High-sensitivity diagnosis of AA amyloidosis using Congo red and immunohistochemistry detects missed amyloid deposits. J Histochem Cytochem 1995;43:863-869.

17 Clement CG, Truong LD: An evaluation of Congo red fluorescence for the diagnosis of amyloidosis. Hum Pathol 2014;45:17661772. 